\title{
3DCLIMBER: A climbing robot for inspection of 3D human made structures
}

\author{
Mahmoud Tavakoli, Ali Marjovi, Lino Marques and Aníbal T. de Almeida
}

\begin{abstract}
DCLIMBER is a running project in the University of Coimbra for developing a climbing robot with the capability of manipulating over 3D human-made structures. This paper mainly discuss the conceptual and detailed design and development of a Pole Climbing robot with minimum degrees of freedom which can climb over 3D structures with bends and branches followed by Preliminary test results of the robot performance. Electronics architecture and control algorithms are briefly described. The paper finishes with discussion of the current results and identifies some future works.
\end{abstract}

\section{INTRODUCTION}

Development of climbing robots was a challenging area during last decade. Different types of climbing robots were developed either for climbing over flat or curved surfaces. For holding robot attached to a smooth surface, suction cups [1], [2], [3], [4] or magnets [5], [6] were used. Robots whose end-effectors match engineered features of the environment like fences or porous materials or bars [7], [8], [9], [10] were developed. Robots for climbing inside pipes or ducts [11], [12] or climbing over poles [13], [14], [15], [16], [17], [18] were also developed. The later group is called Pole Climbing Robots (PCRs). Previously developed PCRs were based on either continuous or step-by-step based climbing mechanisms. Continuous motion PCRs [17], [19] which use tires both for climbing and gripping to the pole are faster and lighter than step-by-step motion PCRs. Their main drawback is the lack of maneuverability. These kinds of robots are mostly appropriate for climbing over simple poles and performing simple tasks which don't need a manipulator, like washing the poles. On the other hand, if one robot aims to perform more complicated tasks, like welding, testing or painting of pipes, a step-by-step based design is a better choice. The reason is that this types of robot takes advantage of its separate gripping module which makes the robot more stable on the pole. Also it has a separate climbing module which can be used for manipulation and performing complicated tasks. The selection of an optimized design highly depends on the application. It is obvious that using a step-by-step mechanism for washing poles is possible but it is not the best solution. Additionally, for step-bystep based design, several configurations for the climbing structure can be considered, namely: serial, parallel or hybrid mechanisms. Each of the mentioned mechanisms have some advantages and disadvantages when compared to another. The climbing configuration is an important issue which is

\footnotetext{
The authors are with the Institute for Systems and Robotics, Department of Electrical and Computer Engineering, University of Coimbra, 3030-290 Coimbra, Portugal. \{mahmoud, ali, lino, adealmeida\}@isr.uc.pt
}

highly related to the considered applications for the robot. R. Saltarn et al. have developed a 6-DOF parallel robot with pneumatic actuators [14] C. Balaguer et al. developed a 6-DOF serial climbing robot for inspection applications in 3D complex environments with $75 \mathrm{~kg}$ of mass [13]. Tavakoli et al. developed a hybrid (serial-parallel) mechanism using electrical cylinders [16]. As pole climbing robots should take their weight up during climbing, it is very important to design optimized and dedicated mechanisms to decrease the weight of the robot. Based on this fact, a designer should consider optimization in all steps of the design process to reduce the weight and the size of the robot as much as possible. The most significant optimization step takes place in the conceptual design step. This means to select the best mechanism with minimum DOFs able to perform the proposed tasks without including redundant capabilities. Redundant DOFs make the robot heavier without necessarily increasing the robot abilities for performing a given task. The aim of the project is performing periodical inspections of the pipes in the industrial plants in order to detect the progression of material degradation and welding defects. To do so a fast rotation around the structure which enables the robot to scan the pole's surface is required. This issue was considered in the design of the 3DCLIMBER robot. A climbing robot which have the ability of climbing from 3D tubular structures, with bends and branches and be able to scan the whole surface of the pipes, may be equipped with NDT probes and do the required inspections automatically. In this paper we address the problem of designing a mechanism with minimum degrees of freedom which can climb over 3D structures with bends and branches. Then we describe the detailed design of the robot and discuss its performance based on test results of the developed prototype.

\section{CONCEPT}

Traveling along a pole or tubular structures with bends and branches requires at least four degrees of freedom [18]. It includes Tz: a translational degree of freedom for motion along the pole axis (Fig. 1), Rz: a rotational DOF for rotation around the pole axis to appropriately align the mechanism with direction of the bend or branch prior to reaching the bend or branch and also to appropriately position or orient the mechanism on the pole for the manipulation task at hand (Fig. 2), Rx: a secondary rotational DOF for rotation around a radial direction of the pole (to orient the gripper perpendicular to the bend section of the pole) and Ty: a translational DOF along the radial direction of the pole (to move the gripper in the direction of the bend section) or 

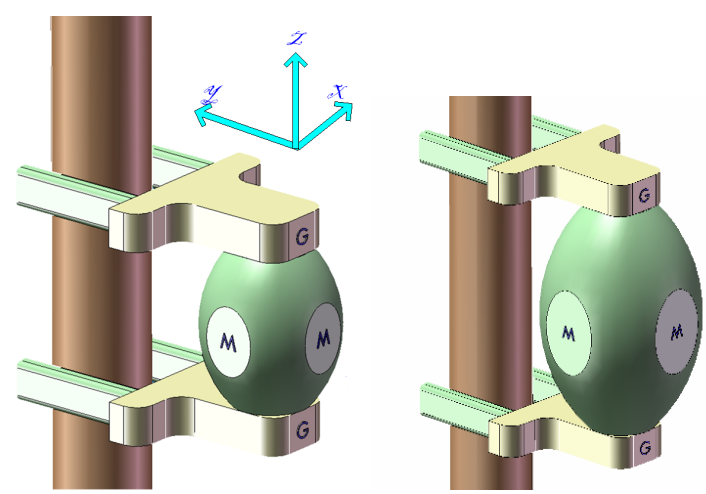

Fig. 1. Climbing along a pole.
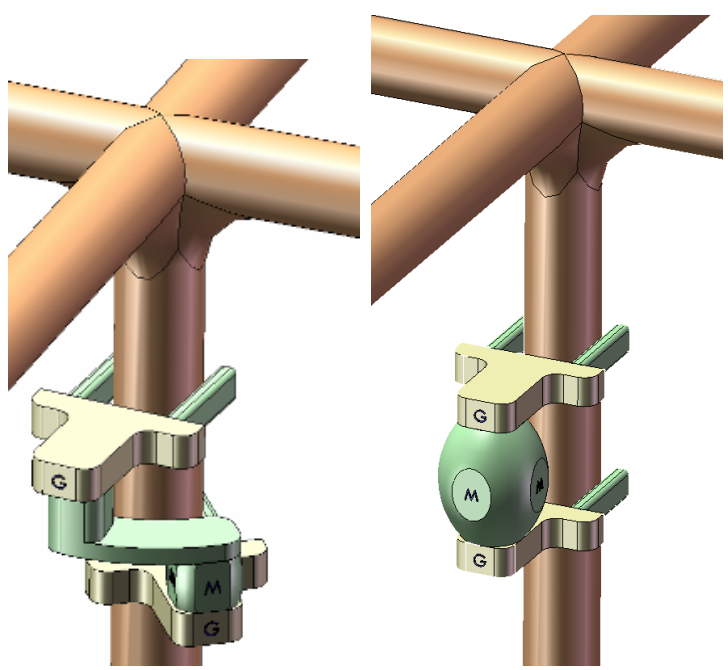

Fig. 2. Rotating around a pole.

to perform the necessary manipulation tasks. The combined action of the latter $2 \mathrm{DOF}$ is needed to re-grasp the pole after the bend / branch (Fig. 3). The combination of the above 4 DOF provides the necessary manipulability to perform necessary operations after reaching target point on pole (i.e. repair, maintenance or even manufacturing operations such as welding). In Figures 1 to 3, "G" stands for gripper and "M" stands for Mechanism. Also the coordinate system axes are changed in figure (Fig. 3), as the working plane of the manipulator is changed. Design and validation of this mechanism is presented in the next section.

\section{Climbing Structure Design And analysis}

\section{A. Design}

A dedicated serial mechanism providing the required DOFs, which was stated in previous section, was designed. The advantages and disadvantage of using serial, parallel and hybrid mechanism for climbing structure depends on the desired applications and was extensively discussed in [20]. It was also discussed why serial mechanisms are the most suitable for our application, while parallel or hybrid mechanisms may be suitable for other climbing applications.

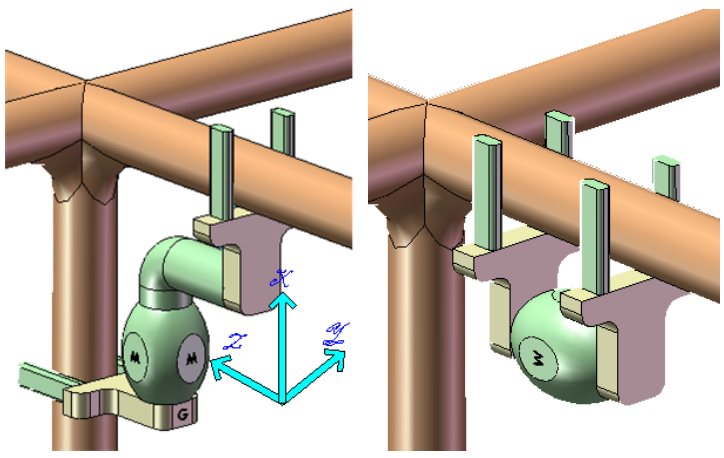

Fig. 3. Overtaking a bend section.

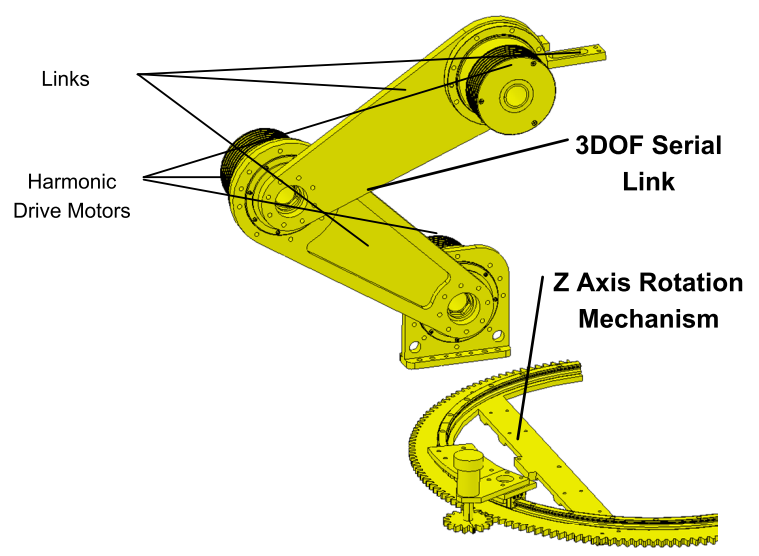

Fig. 4. 4 DOF Climbing structure

The most important reason for choosing serial configuration as climbing mechanism is to increase workspace and manipulability which is a key factor for a multi purpose robot. The designed climbing module consists of a 3-DOF planar Serial arm and a $\mathrm{Z}$ axis rotating mechanism (see Figure 4). Combining the 3-DOF arm with the rotating mechanism provides two rotations $(\mathrm{Rz}$ and $\mathrm{Ry})$ and two translations (Tx and $\mathrm{Tz}$ ) on the manipulator in relation with the base, which are necessary to achieve the design objectives as explained previously. The rotating mechanism which is designed in a different way from traditional serial arms, not only is necessary for orienting the robot for appropriate bend section but also to significantly increase the manipulability of the robot as in this case the robot can rotate around the pole axis and scan the surface of the pole. It also increases significantly the workspace of the robot.

\section{B. Kinematics, dynamics and workspace analysis}

In order to calculate length of the links and required torque for each joint and to select the appropriate actuators, kinematics, dynamics and workspace analysis were performed using standard techniques [21] and are briefly described here to clarify the design process. Figure (5) shows a simplified model of the serial 4 DOF mechanism. In this model the rotational guide is replaced with a simple link 


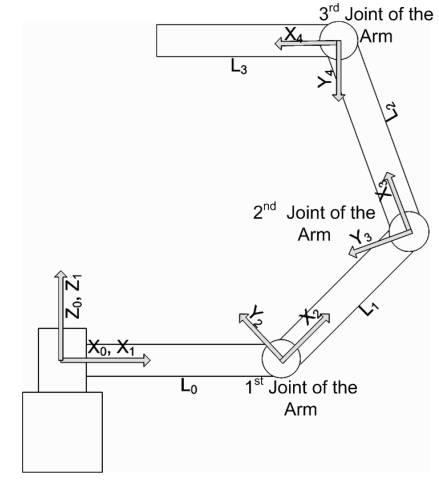

Fig. 5. Simplified model of the 4 DOF mechanism.

in order to simplify the kinematics and workspace analysis. Using this model, direct and inverse kinematics equations, Jacobean matrix and dynamic equations were obtained. Then the required workspace which makes the mechanism be able to pass the bend sections up to $90{ }^{\circ}$ were calculated. Afterward length of the links were selected in order to cover the required workspace. Considering the link lengths and dynamic analysis equations, the required torque for each joint was calculated and appropriate actuators for each joint were selected. To validate the design, the robot model were developed in Solidworks and simulated in Cosmos motion package. Some routines were developed to generate trajectories for each joint so that the robot travel along a straight line or pass a bend section with a specific bend angle. Then the generated trajectories were used by the simulation engine. Simulations showed that the robot is able to pass the bend section of 90 degree with the calculated link lengths and actuators. Figure 6 shows some shots from simulation of the robot model.

\section{Detailed Design, Assembly and Control}

The proposed PCR consists of two main parts: the 4DOF climbing module and the gripping modules. One of the grippers is attached to a manipulator, and the other one is attached to the base of a rotating platform. This configuration provides four DOFs between grippers, allowing the movement along poles with different cross sections and geometric configurations. The proposed design takes advantage of novelties in the design of both climbing and gripping modules. Weight optimization was considered in the design of all of the robot's non standard parts as the weight is a very important factor in climbing robots. All non standard parts were designed and manufactured with 7075T6 aluminium, which has good mechanical properties. This type of aluminum is heavily alloyed with zinc making it very tough and strong. It has an ultimate tensile strength of 510 $538 \mathrm{MPa}$ which has a very good strength to weight property. More information on robot materials can be found in [22].

\section{A. Grippers}

Each gripper consists of two unique multi-fingered Vshaped bodies, a brushless motor, one right hand and one

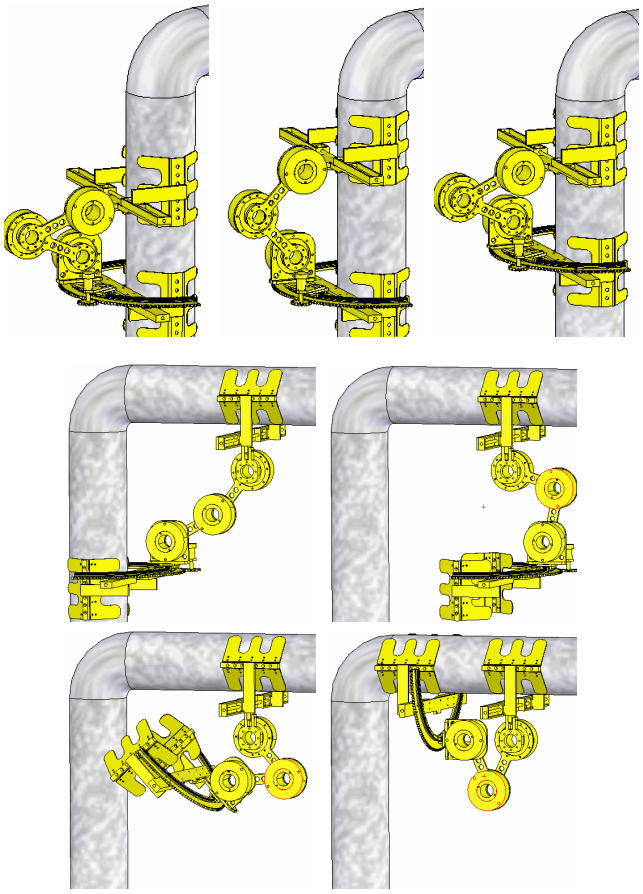

Fig. 6. Sample shots of simulated motion of the robot model.

left hand ball screws and 2 linear guides. V shaped grippers have mechanical self centering properties which significantly reduce the control efforts to precisely control the position of robot, in order to perform safe gripping (Fig. 7). V-Shaped part of the gripper is designed long enough $(250 \mathrm{~mm})$, so that each gripper can withstand the total torque which is generated by the robot weight and by the motors reaction torques. Therefore when one of the grippers is attached to the pole the other gripper can manipulate over the pole and perform some tests on the structure. This eliminates the need for an extra manipulating arm and therefore significantly increase the maneuverability of the robot. The contacting part of the gripper is covered with rubber in order to increase the friction between the pole and the gripper thus increasing the safety. Two types of sensors were used in the gripper. Eight Force Sensitive Resistors (FSR) were attached to each gripper in order to measure force on different locations of the gripper, which not only provides information about the amount of force exerted by grippers, but also provide information on how grippers are connected to the structure (Figure 8). If during the operation of the robot, the 3DOF link have some performance error, or if the angle on the bend section of the robot is not exactly the one expected, the gripper will not oriented exactly perpendicular to the pole. In this case different FSR sensors of the gripper report different values which means incorrect orientation of the gripper. In case of happening, an error will be reported to the user for further decision.

Another advantage of FSR sensors was during the assembly and calibration of the gripper. As both links of each gripper should be installed completely symmetric to the 


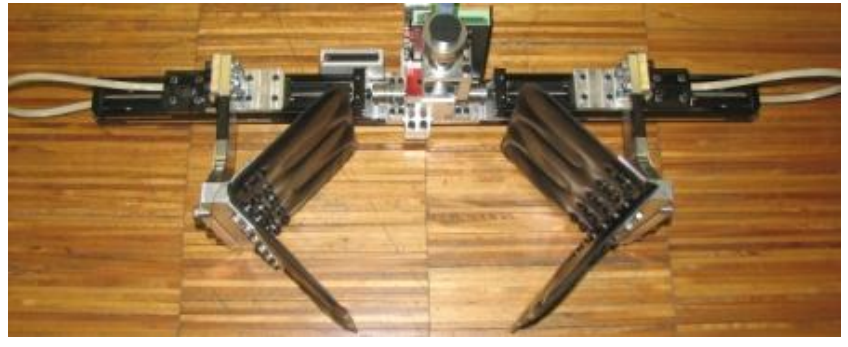

Fig. 7. Gripper of the 3DCLIMBER robot

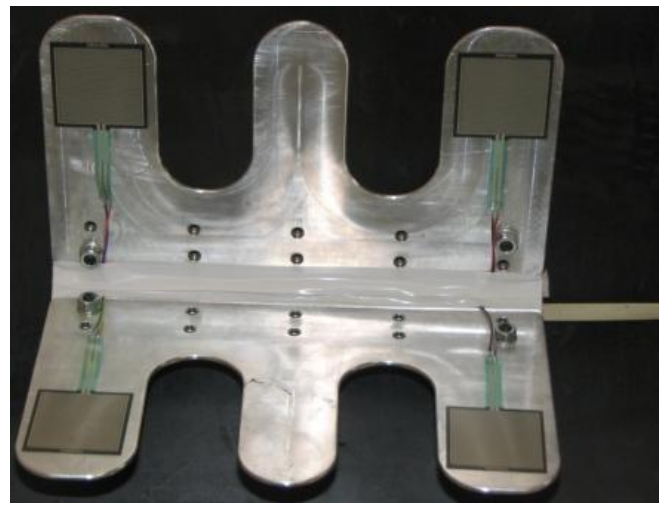

Fig. 8. FSR sensors attached to a gripper

center, measured values from the FSRs helped us to precisely install the grippers.

On the other hand, as FSR sensors are covered by compressible rubber and are not contacting the structure directly, experienced lack of precision on their values. To overcome this problem two strain gauge were glued to each link of the gripper. When the gripper grasps the structure, both links bend a little, which are measured by the strain gauges and consequently the force applied by the gripper is calculated. When the force reaches the desired value, the gripper's motor will stay powered on the current ampere. This is done automatically by the control software. Each gripper is actuated by a 50 Watt Maxon brushless DC motor coupled with a planetary gearbox which can apply $5 N . m$ torque. Coupled by THK ballscrew with $2 \mathrm{~mm}$ pitch the gripper can exert forces of $1000 \mathrm{~N}$. When the gripper is opening, the motor is controlled with position control. When the gripper is closing torque control is applied to the motor in order to control the amount of the force applied by gripper. To increase the safety, the gearbox ratio and ball screw pitch are calculated in a way that after the gripper grasp the structure, the robot can stay attached to the pole by one gripper even if there is a power failure. This was successfully tested. But during the robot operation, after the gripper grasps the structure, the motor will still remain powered and apply torque to increase safety.

\section{B. Climbing structure}

The climbing structure previously described was implemented with the following elements:

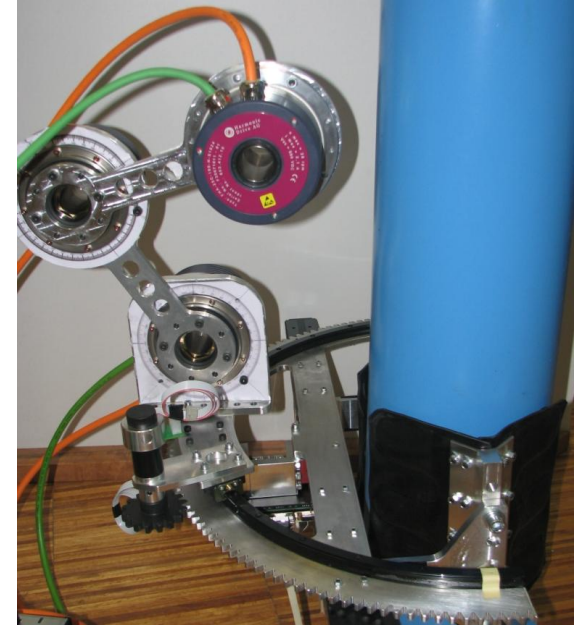

Fig. 9. 4DOF climbing mechanism

TABLE I

MAIN RoBot CHARACTERISTICS

\begin{tabular}{|c|c|}
\hline Degrees of Freedom & 4 \\
\hline Quantity of Motors & 6 \\
\hline Climbing Procedure & Step by Step \\
\hline Weight $(\mathrm{kg})$ & 42 \\
\hline Material of the Parts & Aluminium 7075-T6 \\
\hline Robot Size $(\mathrm{m})$ & $0.5 \times 0.6 \times 0.5$ \\
\hline Extended Robot Size $(\mathrm{m})$ & $0.5 \times 0.6 \times 1$ \\
\hline Climbing Speed $(\mathrm{m} / \mathrm{min})$ & 1 \\
\hline
\end{tabular}

- The 3DOF serial link includes 3 Harmonic Drives AC brushless motors coupled with 160 to 1 Harmonic Drive gearbox, capable of generating torques up to 260 N.m. Harmonicdrive gearboxes are lighter, more precise and more efficient than other types of gearboxes . Figure 9 shows the 4DOF climbing structure.

- The rotation mechanism around the axis of the structure which consists of a THK rotation guide and slider, gearing mate and a Maxon brushless DC motor. This rotation mechanisms provides a fast manipulation around the structure axis, which is necessary for performing most of the inspection tasks like inspection of welding. Table I shows the main characteristics of the robot.

\section{Electronics architecture and control}

As illustrated in Figure 10, three AC brushless motor controllers, three DC brushless motor controllers and a data acquisition module are used. AC motor drivers are SEW 3phase $1.4 \mathrm{KW}$ driver with CANopen interface. DC motor drivers are fully digital intelligent servo drives from TECHNOSOFT with CANopen interface. Controllers can control motors in position, velocity and torque control modes. All motors of the climbing structure( Three AC motors and one $\mathrm{DC}$ motor for the $\mathrm{Z}$ axis rotation) are controlled in position mode. Other 2 DC motors which are used in grippers are controlled in torque control mode. All of the drivers are communicating by CAN bus with CANopen protocol. A 


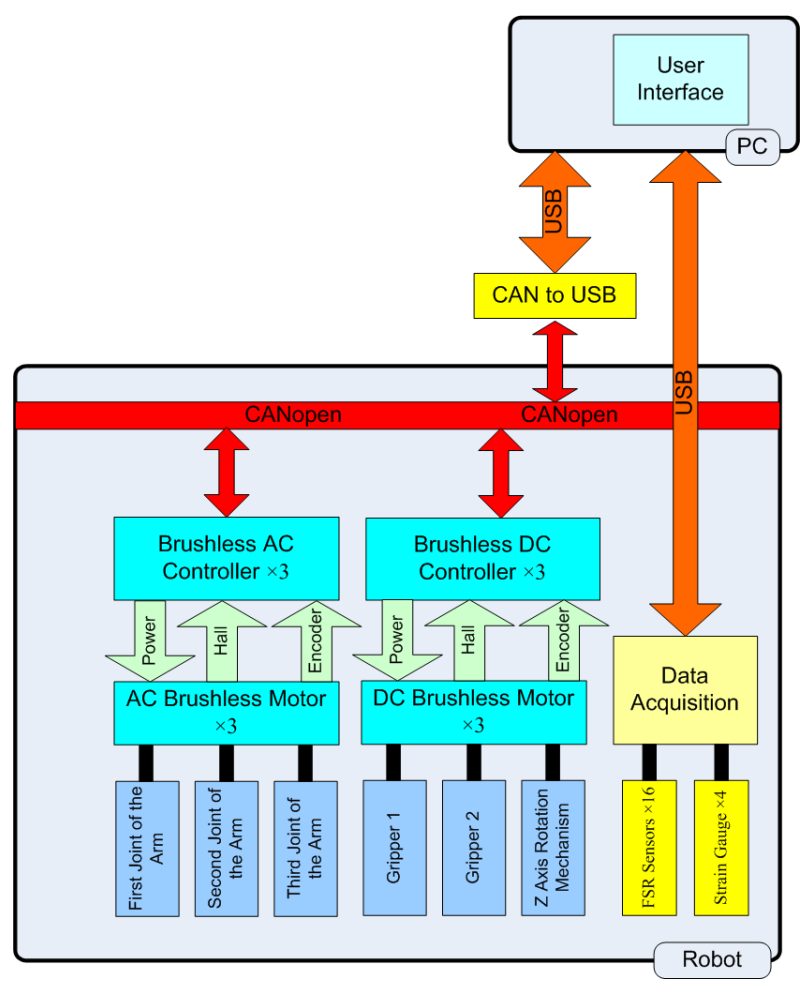

Fig. 10. Electronics architecture of the 3DCLIMBER robot

CAN to USB module is used to connect the CANbus to PC. Data acquisition modules are from National Instruments. Each module provides connection to eight analog input (AI) channels with USB interface. A user interface and an upper level communicating software have been developed in Visual $\mathrm{C} \sharp$.NET in which user can communicate with the robot and set some parameters. Parameters include geometry of the structure, current position of joints, climbing velocity and amount of force which should be exerted by grippers. The user interface also demonstrates a simplified model of the robot, in which user can see the current position of the robot in structure in real time. The upper level controlling program then generates trajectories in joint space for all motors of the climbing structure and generates contol commands for grippers and and send them to the CANbus. It also checks the data from the sensors to ensure safe gripping.

\section{Automation}

At the current stage, the user sends the high level control commands to the control algorithm. The command can be "Step Forward", "Step Backward", "Pass Bend" etc. Then the control algorithm generates and sends the low level control commands to the actuators. For instance a "Step Forward" command includes opening of the upper gripper, moving up the manipulator in a straight line, closing the upper gripper, opening the lower gripper, moving up the lower gripper and closing the lower gripper. Straight line manipulator trajectories are generated by the control algorithm using the Taylor method [23]. Currently we are integrating

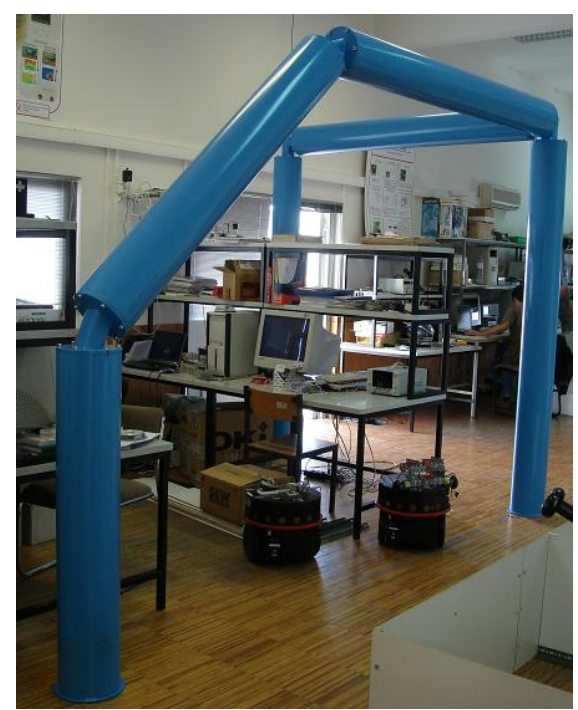

Fig. 11. Developed structure for test of the pole climbing robot.

optical triangulation and ultrasonic trilateration for absolute localization of the robot on the structure, and also higher level control algorithms in order to automate the climbing process of the robot on the structure.

\section{EXPERIMENTS AND RESULT}

A structure was developed as a test environment of the robot which includes bends of 45 and 90 degrees and a multi branch section (Figure 11). The structure have a diameter of $219 \mathrm{~mm}$. The robot was successfully tested on the structure. It successfully passed bends of $45^{\circ}$ and $90^{\circ}$. Figure 13 shows the robot climbing over the structure.

Also a problem was revealed during the experiments, which we are currently working to address it. Our preliminary experiments showed that shortly after a gripper grasps the pole, it tends changing its tilt angle. This is due to the torques resulted by the weight of the robot. Therefore the closed gripper does not stay perpendicular to the pole until end of the step (Figure 12b). Consequently as the other gripper maintains the $180 \mathrm{deg}$ with the first gripper, it will not be perpendicular to the pole. Therefore a perfect gripping action can not be established. It should be noted that it is usually a small error, but it accumulates in each step. To compensate this error we are working on integration of an accelerometer on each gripper to measure the tilt angle of the gripper. After achieving each step, the 3DOFs arm will compensate the error so that the open gripper will stay perpendicular to the structure. Figure 12 shows an exaggerated representation of the problem and the proposed compensation method.

\section{CONCLUSIONS AND FUTURE WORKS}

Climbing and manipulation along 3D structures with bends and branches requires at least 4 DOFs. Therefore, a 4-DOF serial mechanism was designed and developed as climbing structure of the 3DCLIMBER robot. Using the results from kinematics and workspace analysis, the detailed design of 

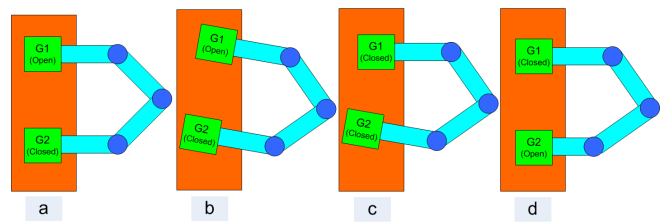

Fig. 12. Demonstration of the tilt angle error and compensation. a-Correct status. b-After occurrence of the error. c- Error compensation for the upper gripper. d-Error compensation for the lower gripper.

the robot was achieved and then validated by simulating movement of the 3D model of the robot along a typical pole. The robot was developed and successfully tested. In this step mostly commercial drivers and boards were utilized to drive actuators, read sensor data and control the robot.

Future works include using multi-criteria optimization techniques to optimize length of the links and to optimize the trajectories, integration of triangulation and trilateration sensors for absolute localization of the robot, assembly of welding test probes and test of the robot against a specified task like scanning the structure to locate defective welding areas.
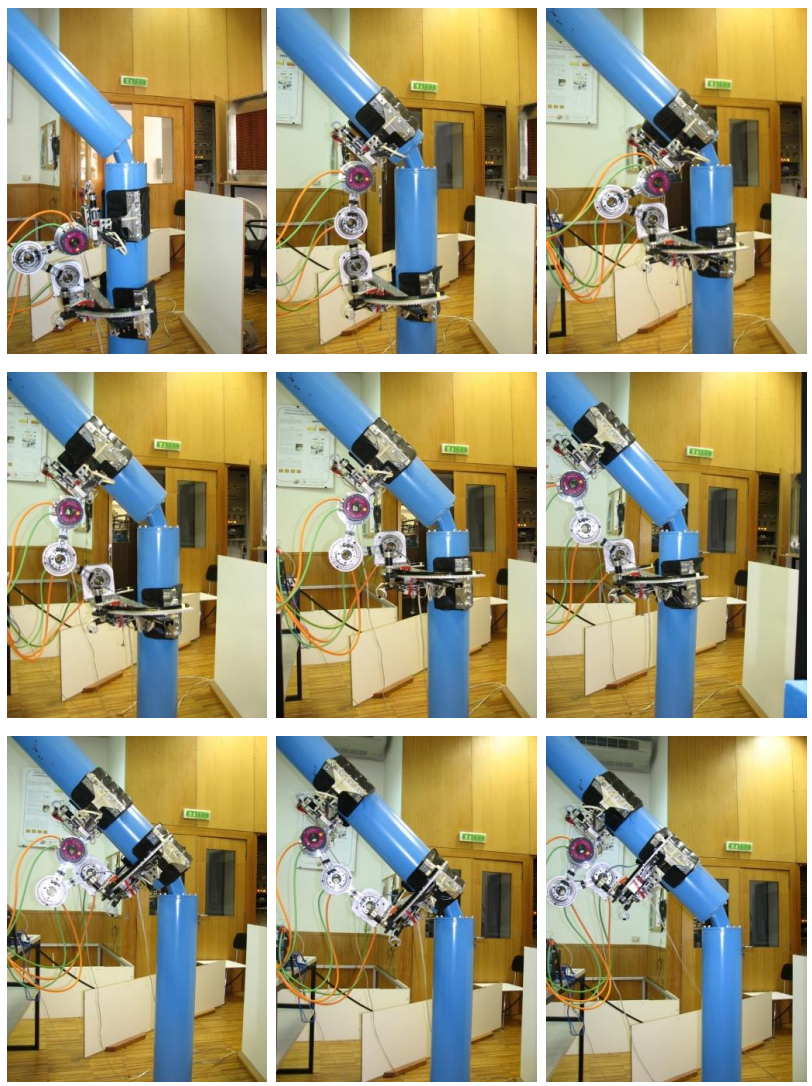

Fig. 13. Sample shots of the experimental results.

\section{REFERENCES}

[1] H. Dulimarta and R. L. Tummala, "Design and control of miniature climbing robots with nonholonomic constraints," in World Congress on
Intelligent Control and Automation, Shanghai, P.R.China, June 2002.

[2] A. Nagakubo and S. Hirose, "Walking and running of the quadruped wall climbing robot," in Proc. IEEE Int. Conf. on Robotics and Automation, San Diego, CA, USA, May 1994, pp. 1005-1012.

[3] S. W. Ryu, J. J. Park, S. M. Ryew, and H. R. Choi, "Self-contained wall-climbing robot with closed link mechanism," in IEEE/RSJ Int. Conf. on Intelligent Robots and Systems, Hawai, USA, Oct-Nov 2001, pp. 839-844.

[4] L. S. W. Yan, X. Dianguo, Z. Yanzheng, S. Haoand, and G. Xuesban, "Development and application of wall-climbing robots," in Proc. IEEE Int. Conf. on Robotics and Automation, Detroit, USA, May 1999, pp. 1207-1212.

[5] J. C., M. Prieto, M. Armada, , and P. G. de Santos, "A six-legged climbing robot for high payloads," in IEEE Int. Conf. on Cont. App, Trieste, Italy, Sept. 1998, pp. 446-450.

[6] S. Hirose, A. Nagabuko, and R. Toyama, "Machine that can walk and climb on floors, walls, and ceilings," in Proc. IEEE Int. Conf. on Advanced Robotics, Pisa, Italy, June 1991, pp. 753-758.

[7] D. Bevly, S. Dubowsky, and C. Mavroidis, "A simplified cartesiancomputed torque controller for highly geared systems and its application to an experimental climbing robot," ASME J. of Dynamic Systems, Measurement, and Control, no. 122(1), pp. 27-32, 2000.

[8] Y. Xu, H. Brown, M. Friendman, and T. Kanade, "Control system of the self mobile space manipulator," IEEE Trans. on Control Sys. Technology, vol. 2, no. 3, pp. 207-219, 1994

[9] M. Yim, S. Homans, and K. Roufas, "Climbing with snake-robots," in IFAC Workshop on Mobile Robot Technology, Jejudo, Korea, 2001.

[10] H. Amano, K. Osuka, and T.-J. Tarn, "Development of vertically moving robot with gripping handrails for fire fighting," in IEEE/RSJ Int. Conf. on Intelligent Robots and Systems, Maui, USA, Oct-Nov 2001, pp. 661-667.

[11] W. Neubauer, "A spider-like robot that climbs vertically in ducts or pipes," in IEEE/RSJ Int. Conf. on Intelligent Robots and Systems, Munich, 1994, pp. 1178-1185.

[12] T. Rossmann and F. Pfeiffer, "Control of an eight legged pipe crawling robot," in Int. Symp. on Experimental Robotics, Barcelona, 1997, pp. 353-346.

[13] C. Balaguer, A. Gimenez, J. Pastor, V. Padrón, and M. Abderrahim, "A climbing autonomous robot for inspection applications in $3 \mathrm{~d}$ complex environments," Robotica, vol. 18, pp. 287-297, 2000.

[14] M. Almonacid, R. Saltaren, R. Aracil, and O. Reinoso, "Motion planning of a climbing parallel robot," IEEE Transactions on Robotics and Automation, vol. 19, no. 3, pp. 485-489, 2003.

[15] Z. Ripin, T. B. Soon, A. Abdullah, and Z. Samad, "Development of a low-cost modular pole climbing robot," in TENCON, Kuala Lumpur, Malaysia, 2000, pp. 196-200.

[16] M. Tavakoli, M. Zakerzadeh, G. Vossoughi, and S. Bagheri, "A hybrid pole climbing and manipulating robot with minimum DOFs for construction and service applications," Journal of Industrial Robot, vol. 32, no. 2, pp. 171-178, March 2005.

[17] A. Baghani, M. Ahmadabadi, and A. Harati, "Kinematics modelling of a wheel-based pole climbing robot (UT-PCR)," in Proc. IEEE Int Conf. on Robotics and Automation, Barcelona, April 18-22 2005.

[18] G. Vossoughi, S. Bagheri, M. Tavakoli, M. Zakerzadeh, and M. Houseinzadeh, "Design, modeling and kinematics analysis of a novel serial/parallel pole climbing and manipulating robot," 7th Biennial ASME Engineering Systems Design and Analysis conference, Manchester, pp. 19-22, 2004.

[19] H. Hosokai and F. Hara, "Manoeuvrability passing over obstacles on a pipeline by a pipeline inspection," in CLAWAR, Karlsruhe, Germany, 2001.

[20] M. Tavakoli, L. Marques, and A. de Almeida, "Pole climbing and manipulating robots: Assessment of different design categories," in Proc. 37th Intl. Symp. on Robotics, Munich, Germany, May 2006.

[21] J. Craig, Introduction to Robotics: Mechanics and Control. AddisonWesley Longman Publishing Co., Inc. Boston, MA, USA, 1989.

[22] Robotbooks, "Robot materials," http://www.robotbooks.com/robotmaterials.htm, june 2008 .

[23] R. Taylor, "Planning and execution of straight line manipulator trajectories," IBM Journal of Research and Development, vol. 23, no. 4, pp. 424-436, 1979. 